\title{
Resequencing and genome-wide association studies of autotetraploid potato
}

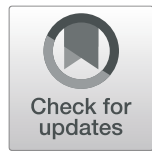

Feng Zhang ${ }^{1 \dagger}$, $\mathrm{Li} \mathrm{Qu}^{2+}$, Yincong Gu${ }^{3}$, Zhi-Hong Xu ${ }^{4}$ and Hong-Wei Xue $2,4^{*}$ (D)

\begin{abstract}
Potato is the fourth most important food crop in the world. Although with a long history for breeding approaches, genomic information and association between genes and agronomic traits remain largely unknown particularly in autotetraploid potato cultivars, which limit the molecular breeding progression. By resequencing the genome of 108 main cultivar potato accessions with rich genetic diversity and population structure from International Potato Center, with approximate 20-fold coverage, we revealed more than 27 million Single Nucleotide Polymorphisms and $\sim 3$ million Insertion and Deletions with high quality and accuracy. Domestication analysis and genome-wide association studies (GWAS) identified candidate loci related to photoperiodic flowering time and temperature sensitivity as well as disease resistance, providing informative insights into the selection and domestication of cultivar potato. In addition, GWAS with GWASploy for 25 agronomic traits identified candidate loci by association signals, especially those related to tuber size, small-sized tuber weight and tuber thickness that was also validated by transcriptome analysis. Our study provides a valuable resource that facilitates the elucidation of domestication process as well as the genetic studies and agronomic improvement of autotetraploid potato.
\end{abstract}

Keywords: Solanum tuberosum (potato), Autotetraploid cultivars, Genome-wide association study (GWAS), Domestication, Genome, Resequencing

\section{Core}

Seletive interval analysis combined GWAS of autotetraploid potato identified candidate loci under domestication and associated with agronomic traits, providing informative insights for elucidating the domestication history and selective process of cultivar potato and facilitating the genetic studies and agronomic improvement of autotetraploid cultivar potato.

\footnotetext{
* Correspondence: hwxue@sjtu.edu.cn

${ }^{+}$Feng Zhang and Li Qu contributed equally to this work.

${ }^{2}$ Shanghai Collaborative Innovation Center of Agri-Seeds, Joint Center for Single Cell Biology, School of Agriculture and Biology, Shanghai Jiao Tong University, Shanghai 200240, China

${ }^{4}$ National Key Laboratory of Plant Molecular Genetics, CAS Center for Excellence in Molecular Plant Sciences, Chinese Academy of Sciences, Shanghai 200032, China

Full list of author information is available at the end of the article
}

\section{Introduction}

Potato (Solanum tuberosum L.) is the predominant vegetable crop in the global food system and also a critical food crop (Birch et al. 2012). Cultivated potatoes with autotetraploid inheritance have been domesticated from diploid wild species native to the Andes of Southern Peru (Spooner et al. 2005), and improved by domestication and modern breeding in many aspects including yield, quality and disease resistance. Consumer acceptance and distinct environmental conditions are the main reason for constrained spread of potatoes, and the domestication dispersal of crops outside of their native range need extensive adaptation to the new environments moved along latitudinal gradients (Diamond, 2002; Shennan et al. 2013). Elucidating the selective gene loci will surely help to understand the domestication of potato and relation between climate and human

C C The Author(s). 2022 Open Access This article is licensed under a Creative Commons Attribution 4.0 International License, which permits use, sharing, adaptation, distribution and reproduction in any medium or format, as long as you give appropriate credit to the original author(s) and the source, provide a link to the Creative Commons licence, and indicate if changes were made. The images or other third party material in this article are included in the article's Creative Commons licence, unless indicated otherwise in a credit line to the material. If material is not included in the article's Creative Commons licence and your intended use is not permitted by statutory regulation or exceeds the permitted use, you will need to obtain permission directly from the copyright holder. To view a copy of this licence, visit http://creativecommons.org/licenses/by/4.0/ The Creative Commons Public Domain Dedication waiver (http://creativecommons.org/publicdomain/zero/1.0/) applies to the data made available in this article, unless otherwise stated in a credit line to the data. 
selection. However, the selective genes and domestication histories for cultivars are poorly understood yet.

Traditional breeding of potato is often faced with various difficulties. Considering the long breeding cycles and low selection efficiency, there is an urgent need to develop molecular approaches to facilitate the breeding efficiency. Although several molecular marker techniques including simple sequence repeat (SSR) and Expressed sequence tag-SSR (EST-SSR) have been applied to assess the genetic diversity, germplasm identification and population structure (Zhu et al. 2008; Rosyara et al. 2016; Zhao et al. 2017), however, the complexity of autotetraploid genome and insufficient genomic information are still big obstacles to functional research and molecular breeding (Levy and Veilleux, 2007). Autotetraploid cultivars are grown most widely all over the world and also the main materials used for cross breeding (Zhang et al. 2019), deciphering the genome sequence and analysis of trait diversity and genomic association is indispensable.

Several studies have sequenced some representative potato samples and mainly analyzed the sequence diversity to reveal the complex evolutionary history of adaptation to environments (Gutaker et al. 2019) and impact of domestication on genome diversity, and identified key loci selected for cultivation (Hardigan et al. 2017) or some molecular markers (Li et al. 2018). Recent advances in next-generation sequencing (NGS) and increasing capacity of computational analyses of massive data enabled us to comprehensively analyze the whole genome of autotetraploid potato cultivars with whole-genome sequencing (WGS). Indeed, genomewide association study (GWAS) has been applied to discover causal variants for complex traits effectively with high resolution at genome level comparing with the traditional linkage mapping strategy (Zhu et al. 2008). Combination of WGS and GWAS has been shown as an effective method for identifying genome-phenotype associations in many crop species including rice (Huang et al. 2012), sorghum (Tao et al. 2020) and maize (Li et al. 2019), which provides informative genomic resources for functional studies. Nevertheless, the study is still very preliminary in potato especially with combination of WGS and GWAS.

The GWAS of autotetraploid potato is rare and difficult, mainly due to the lack of genomic information and accurate assembled sequence of autotetraploid potato as a reference. Fortunately, construction of a chromosome-scale long-read reference genome assembly enabled the GWAS of autotetraploid potato (Pham et al. 2020), which is an updated version of the DM1-3516 R44 genome sequence, a doubled monoploid clone of $S$. tuberosum Group Phureja using a whole-genome shotgun sequencing approach with shortread sequence data (Xu et al. 2011). Hence combination of WGS and GWAS as well as a chromosome-scale long-read reference genome make it possible to explore more genomic resources for cultivated autotetraploid potato.
In this study, we resequenced 108 core cultivated germplasms of autotetraploid potato from International Potato Center by whole-genome sequencing and revealed the loci associated with the rich genetic diversity by analyzing 25 agronomic traits with GWASploy analysis. One hundred and thirty-eight high-confidence selective sweeps comprising 54 predicted genes were dissected by domestication analysis, which is beneficial for investigating the diversity and domestication of cultivated potato. In addition, large scale GWAS studies identified 50 candidate loci associating with 15 agronomic traits. These results provide helpful and important genomic resources of cultivated potato and significantly contribute the potato biology and breeding approaches.

\section{Results}

\section{Whole-genome sequencing of $108 \mathrm{CIP}$ and identification of SNPs and InDels}

In this study, 108 high-generation accessions from International Potato Center (designated as CIP accessions, Table S1) were applied for whole-genome sequencing and further analysis. Collected samples were used to construct libraries and genotyped with approximate 20fold-coverage genome sequencing using a barcoded multiplex sequencing approach on the Illumina HiSeq PE (Paired-End) 150 base pairs (bp). Raw paired-end reads were trimmed and filtered to obtain the highquality clean data. Trimmed reads were mapped to the $S$. tuberosum group Phureja DM reference genome (Pham et al. 2020; Xu et al. 2011) for alignment, and GATK (McKenna et al. 2010) was used to detect the population SNP (Single Nucleotide Polymorphisms) and InDel (Insertion and Deletion) (Table S2). After filtering and screening, the high quality and accuracy population SNPs and InDels were finally annotated and analyzed.

We finally identified 27,565,997 SNPs and 2,961,770 InDels by analyzing these CIP accessions, which lays a good foundation for the selective interval analysis and GWAS analysis. A total of 1,565,451 SNPs (4.21\%) and 64,080 (1.37\%) InDels were located in the coding regions, among which 60,265 SNPs or InDels showed potentially significant effects, including 21,694 SNPs $(0.058 \%)$ that may affect 2329 genes by causing start codon changes, premature stop codons or elongated transcripts, and 38,571 InDels $(0.832 \%)$ that may lead to a frameshift in 11,132 annotated genes (Table S3).

Previously, 67 genotypes including 20 Wild diploid species, 20 South American landraces, 23 North American cultivars and 4 Outgroups were resequenced (Hardigan et al. 2017), which were used to capture a broad extent of genome variation and their progenitors. We downloaded the resequencing data of these 67 accessions (NCBI database under PRJNA378971, Hardigan 
et al. 2017) and reanalyzed the 62 accessions of them together with the 108 CIP accessions, with a total of 170 accessions (5 of previous analyzed cultivars were included in CIP accessions for deep sequencing). After mapping with the reference genome (DM v6.1, Pham et al. 2020), we identified 22,003,848 SNPs and 1,878,036 InDels with an average of 25.88 SNPs and 2.16 InDels per kb. Detailed analysis showed that a total of $1,538,176$ SNPs (5.06\%) and 52,376 (1.75\%) InDels were located in the coding regions, among which 47,533 are potentially being crucial including 19,007 SNPs (0.06\%) affect 3074 genes by causing start codon changes, premature stop codons or elongated transcripts, and 30,552 (1.02\%) InDels lead to a frameshift in 12,301 annotated genes (Table S4). Considering there are wild diploid species in the analyzed 170 accessions, these data will help the further domestication and selection analysis.

\section{Population structure divergence revealed that CIP accessions are typical cultivated autotetraploid potato}

Population structure is essential to investigate the background of $108 \mathrm{CIP}$ accessions and an important factor for selective interval analysis and GWAS. According to the phylogeny analysis based on SNP data and the principal-component analysis (PCA), the 170 accessions are mainly divided into five categories including Wild, Outgroup, Landrace, reported cultivar and CIP cultivar (Fig. 1A). Analysis showed a mixture between the CIP cultivars and reported ones, confirming that they both are typical cultivated autotetraploid potato with significant genetic differentiation from Wild and Landrace (Fig. 1A, B).

Variation curve of BIC (Bayesian Information Criterion) value showed the optimal $\mathrm{K}$ value, a parameter in $\mathrm{K}$-means clustering used to identify groups and describe the relationship between groups. When $K=2$, the population were divided into two distinct clusters only, Wild species and CIP species, while landrace was differentiated when $K=3$ or higher. Interestingly, from $K=4$, the reported cultivars begun to differentiate from domesticated ones, but a sophisticated genetic structure was observed in CIP cultivars (Fig. 1C, Fig. S1). The differentiation between the reported cultivars and CIP ones may due to that CIP accessions are main cultivated species used for agricultural production with long selection and breeding history than the reported cultivars, and the reported cultivars are mixtures consisting of domestication, improvement, and modern breeding efforts (Hardigan et al. 2017). In addition, differential geographical distribution and genetic distances between them may also result in differentiated population structure considering the relative uniform genetic composition may result from the concentrated geographical distribution (Goulas et al. 2006) and different genetic distances accompanied by unequal wild introgression (Hardigan et al. 2017).

\section{Domestication analysis dissected the selective sweeps across the whole genome}

Domestication of wild potatoes were originated from the Andes of Southern Peru approximately 8000 years ago and were mainly cultivated in altitudes $2000-4000 \mathrm{~m}$ regions that are characterized by short day (SD) condition, high light intensities, and cool temperatures (Zierer et al. 2021). Contemporary autotetraploid cultivars were selected to grow under moderate temperatures and to form tubers under long day (LD) condition later (Zierer et al. 2021). We analyzed the phenotypes of 108 CIP accessions which are main germplasm resources with abundant agronomic traits (these accessions are mainly planted in Yunnan or Gansu province of China). Considering the advantage agronomic traits of autotetraploid cultivar tubers are mainly resulted from human selection, identification of the candidate selected loci will shed light on domestication history and breeding improvement of potato.

To investigate how the genomes of CIP accessions were shaped by environment and human selection, we performed a comprehensive assessment of Wild and CIP population based on genomic analyses of 170 accessions. Selective interval analysis was conducted across the whole genome through a $F_{S T}$ (population-differentiation statistic) and $\theta_{\pi}$ (the $\log 2$ ratio of nucleotide diversity) based cross approach. As a result, a total of 138 with strong selection signals were identified being involved in various physiological processes including photoperiodic flowering, temperature responses, tuber shape and disease resistance (Fig. 2, Table S5), which may be closely correlated to domestication of cultivars. In addition, based on the identified differentiation between the reported cultivars and CIP ones, an XP-CLR analysis across the whole genome was conducted. XP-CLR scores in 20-kb sliding windows revealed the selective regions and genes in the whole genome (Fig. 2, Table S6). We identified many environmental adaptation related regions and genes including those involve in oxidative phosphorylation and peroxisome process, suggesting that different geographical distribution of these two cultivar groups affected their responses to stress and resulted in differential environmental adaptations. In addition, there are differentiations in the metabolism process-related regions, implying the different potato quality selection and breeding measures by human of two cultivars. However, compared with the result of selective interval analysis between Wild and CIP population, there is little overlap of the selective regions and genes between two groups by XP-CLR analysis, which is in agreement with the population structure divergence 


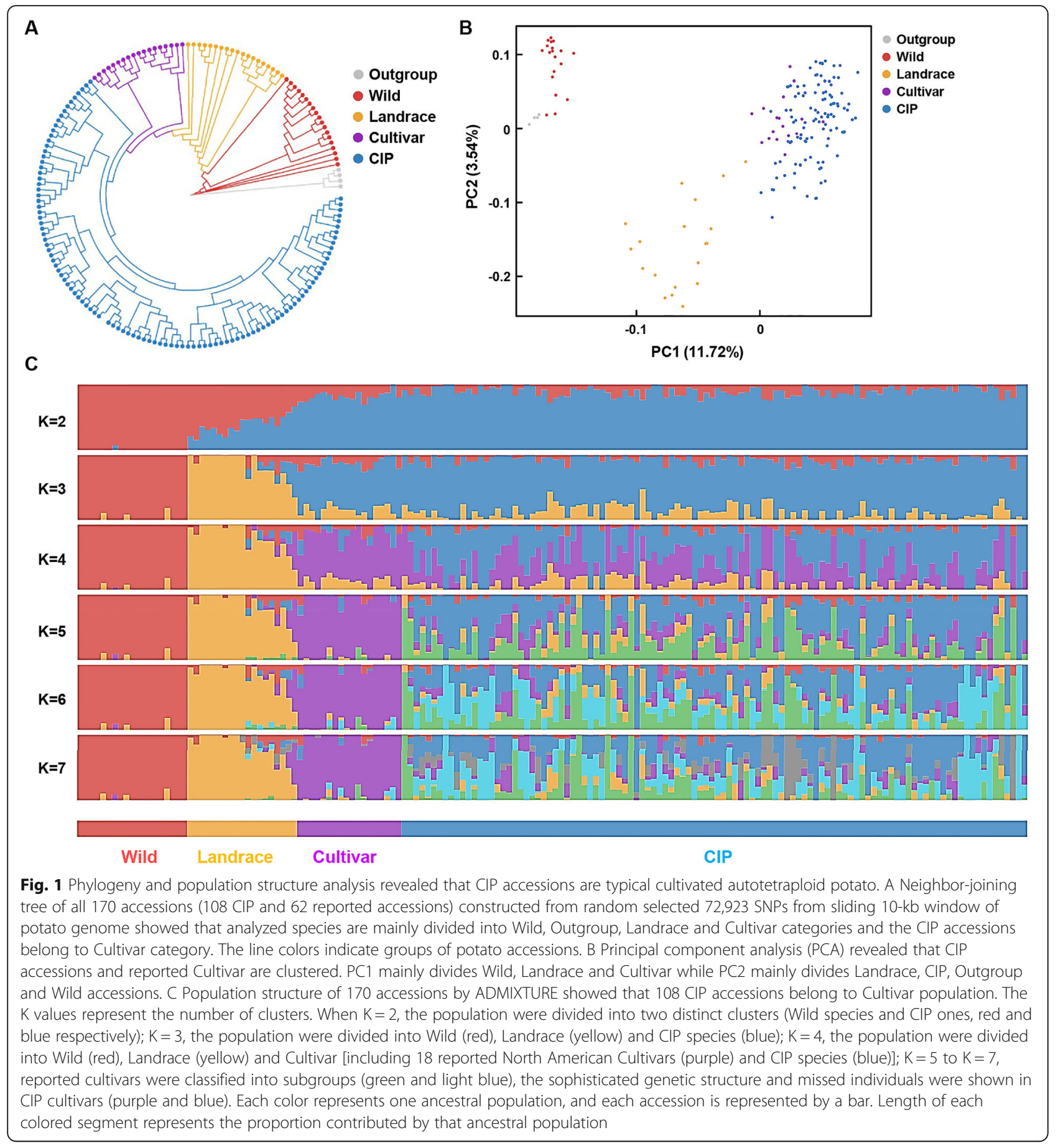

analysis with variation curve of BIC since both the reported cultivars and CIP population are cultivated autotetraploid potato with similar genetic distances. Obviously, the differentiation between the two cultivar groups is smaller than Wild and CIP population on account of the long domestication history and breeding improvement of cultivar groups.

Since geographic expansion along latitudinal gradients required an adjustment to different day length and temperature cues (Fuller and Allaby, 2009), gene loci related to photoperiodic response and temperature sensitivity were analyzed by selective interval analysis (Fig. 2). Analysis reveals that Soltu.DM.10G011640 (homologous to gibberellin oxidase 2, GAox2, in Arabidopsis thaliana, Sang et al. 2020) is under strong selection. Studies have proved that tuberization process relies on both GAdependent and photoperiod-dependent pathways and $\mathrm{GA}$ is required for the stolon meristem elongation 

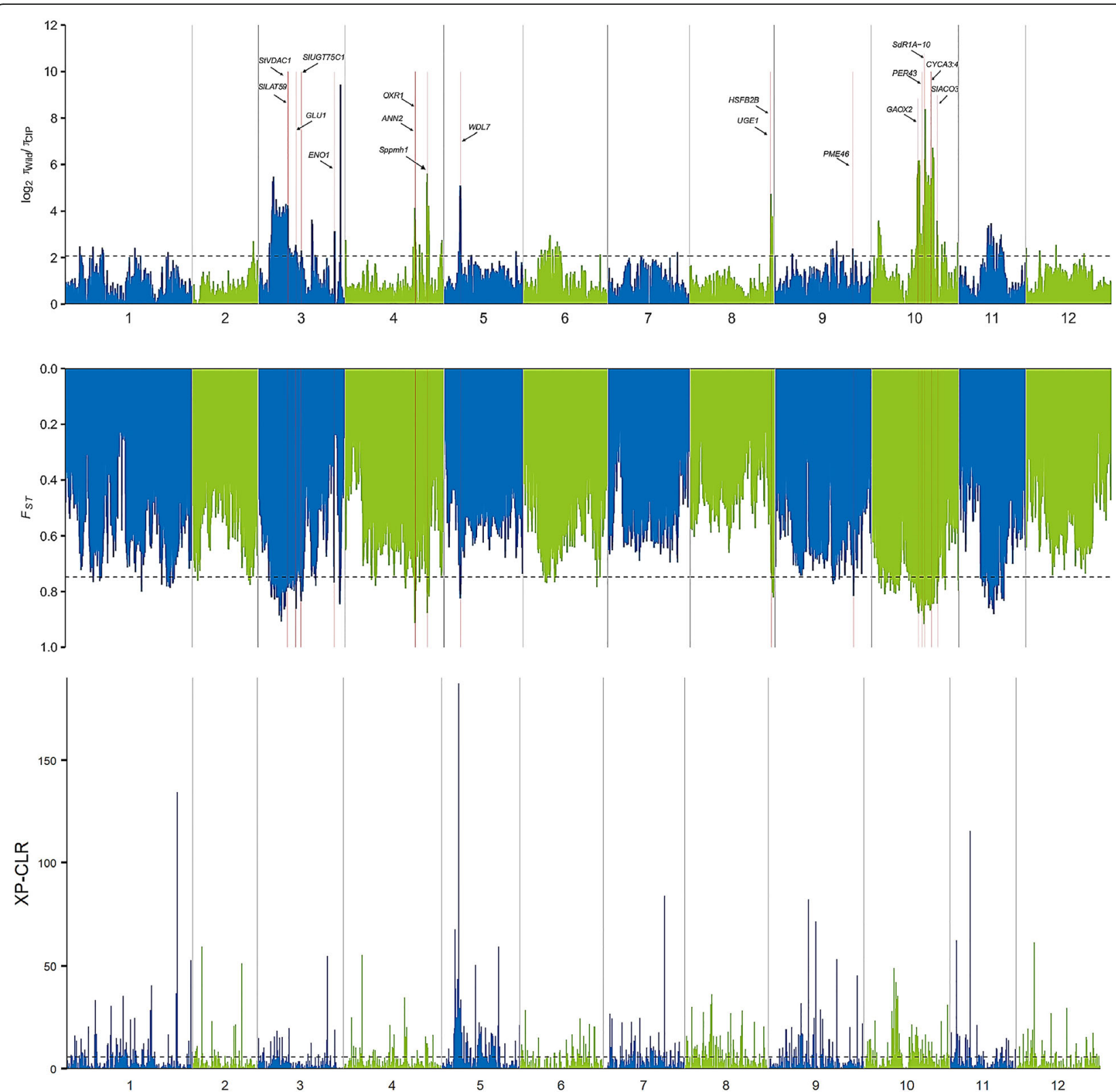

Fig. 2 Selective sweep by genome-wide screening and functional annotations during domestication. Selective sweep regions revealed by the $\log _{2}$ ratio of nucleotide diversity ( $\Pi$, upper), Pairwise fixation index ( $F_{S T}$, middle) of Wild and CIP, and XP-CLR analysis of CIP and reported cultivars (bottom) on 12 chromosomes. Horizontal dashed lines ( $\log _{2} \pi$ Wild $/ \pi C I P=2.07, F S T=0.75$, or XP-CLR $=1 \%$ ) indicate the genome-wide threshold of selection signals. The higher the $\Pi$ value, the greater the difference between two groups. Possible homologous genes of Arabidopsis related to domestication traits mentioned in the text were shown at the selected regions of the whole chromosome (upper). Pairwise fixation index ( $F_{S T}$ ) in 20-kb sliding windows across 12 chromosomes revealed the differentiation degree between Wild and CIP subpopulations. The higher the $F_{S T}$ value, the higher degree of differentiation between two subpopulations (middle). XP-CLR scores in 20-kb sliding windows across 12 chromosomes revealed the differentiation between CIP and reported cultivars in the whole genome. The higher the XP-CLR score, the higher degree of differentiation between CIP and reported cultivars (bottom)

during the tuberization initiation (Martinez-Garcia et al. 2001; Dutt et al. 2017), and selection of GAox2 is agreement with the essential roles of GA and photoperiod in potato tuberization ( $\mathrm{Li}$ et al. 2018).

Potato is grown as an annual crop and non-optimal temperature inhibits the growth and survival and hence reduces the tuber yield and productivity (Levy and Veilleux, 2007). Many wild potato species have suffered cool temperatures while moderate temperatures were selected for contemporary autotetraploid cultivars (Zierer et al. 2021). Some temperature sensitivity related genes of potato under selection were identified, including 
Soltu.DM.04G021330 and Soltu.DM.08G027190, whose homologs of Arabidopsis are ANNAT2 (ANN2, Liao et al. 2017) and HEAT SHOCK TRANSCRIPTION FACTOR B2B (HSFB2B, Charng et al. 2007) respectively, and these genes might be good candidates for breeding improvement of potato in various regions with different temperature preference.

Disease is a big threat to potato yield and quality, and pathogens such as late blight have devastating impacts on potato. Wild potatoes usually possess weaker disease resistance than cultivated potato, and disease resistance is an important agronomic trait under selection analysis. Selective analysis identified some reported potato disease resistant genes including two late blight resistant genes Soltu.DM.10G012850 (R1A-10, Kuang et al. 2005) and Soltu.DM.10G013980 (SbRGA3, Song et al. 2003), which will enlighten the potato breeding.

Cultivated potatoes produce bigger tubers than wild potato during domestication of tuber size. Tuber growth is also crucial for yield and productivity after tuberization and genes correlated with cell division were selected. CYCLIN A3;4 (CYCA3;4) is an important protein controlling formative cell divisions in Arabidopsis (Willems et al. 2020) and its homolog Soltu.DM.10G014530 was identified. In addition, many stress responsive genes are under selection as well, including microtubulelocalized genes Soltu.DM.03G008530 (StVDAC1, Balmer et al. 2004) and Soltu.DM.05G010550 (WDL7 in Arabidopsis, Dou et al. 2021), which play essential roles in mediating stomatal closure in response to drought stress and ABA treatment. Selection of these genes provides informative hints on the environmental adaptation of potato cultivars and related selective loci can serve as the candidate genetic resources for potato improvement.

\section{Correlation analysis revealed the close relationship between agronomic traits of CIP accessions}

International Potato Center created multiple germplasm resources for variety improvement in different countries. Although their genetic background is mixed without obvious subgrouping, the 108 CIP accessions are core cultivars with a rich diversity among agronomic traits, particularly tuber shape and color (Fig. 4A) as well as differential yield and quality traits which are crucial to the selection of varieties. In addition, the difference of tuber color is the result of metabolism variation. We thus detailed analyzed 25 agronomic traits with statistics of these 108 accessions for several successive years, including 9 yield component traits, 10 quality characters and 6 external properties by planting in Gansu province since 2016 (Table S7).

Analysis of the analyzed 25 agronomic traits showed that most of them are normally distributed, indicating they are quantitative traits controlled by polygenes and susceptible to environmental influences. Evaluation by Pearson factor further revealed the close correlations of overall 25 traits quantitatively (Fig. 3). Pearson factor evaluation of yield traits including total commodity rate, commodity rate per plant, commercial potato number, commercial potato weight, small-sized tuber number, small-sized tuber weight, tuber number per plant, yield per plant and plot yield, showed the interrelationship between each two of them. In particular, yield per plant is positively correlated to plot yield and commercial potato weight with high Pearson factor $(0.74$ and 0.97 respectively), indicating the increasing yield per plant is an important means to improve the commercial value of potatoes. All these complex and abundant agronomic traits and their close connections are affected by polygene and environments, which facilitates the genomewide association analysis to identify QTLs.

Small-sized tuber number is positively correlated to small-sized tuber weight and tuber number per plant with high Pearson factor (0.82 and 0.81 respectively, Fig. 4B), suggesting increasing tuber number may also increase tuber weight and both are effective ways to improve yield. External properties include tuber length, tuber width, tuber thickness, tuber aspect ratio, tuber's eye number and the depth of tuber's eye and all of them are crucial for commercial value and consumers' acceptance (Si et al. 2017). Close correlation especially tuber size related traits including length and aspect ratio, tuber thickness and width are detected and will enlighten the potato breeding for the larger market demands (Fig. 4C). In addition, examination of the quality traits of dry matter content including tuber starch, contents of amylose and amylopectin, and contents of various sugar and acid showed the great diversity of these traits, revealing the various levels of quality and flavors in CIP accessions. Evaluation by Pearson factor showed a strong negative correlation between the contents of amylose and amylopectin and a positive association between the contents of glucose and sucrose, suggesting the cross of metabolic pathway such as glycometabolism (Fig. 5). The complex quality traits and close correlations also enable the genome-wide association study to reveal the rich and invaluable genetic resource.

\section{Genome-wide positioned loci associated with key agronomic traits}

The abundant phenotypic diversity without obvious population differentiation in CIP accessions laid good foundation for GWAS positioning. The variation map at singlebase resolution empowered GWAS for 25 agronomic traits in potato. Considering the genome absence of autotetraploid potato and limitation of polyploid GWAS, we applied the GWASpoly to correlate the autotetraploid genotypes for analysis with the data of newly revealed haploid genome (DM v6.1, Pham et al. 2020). In total, 50 


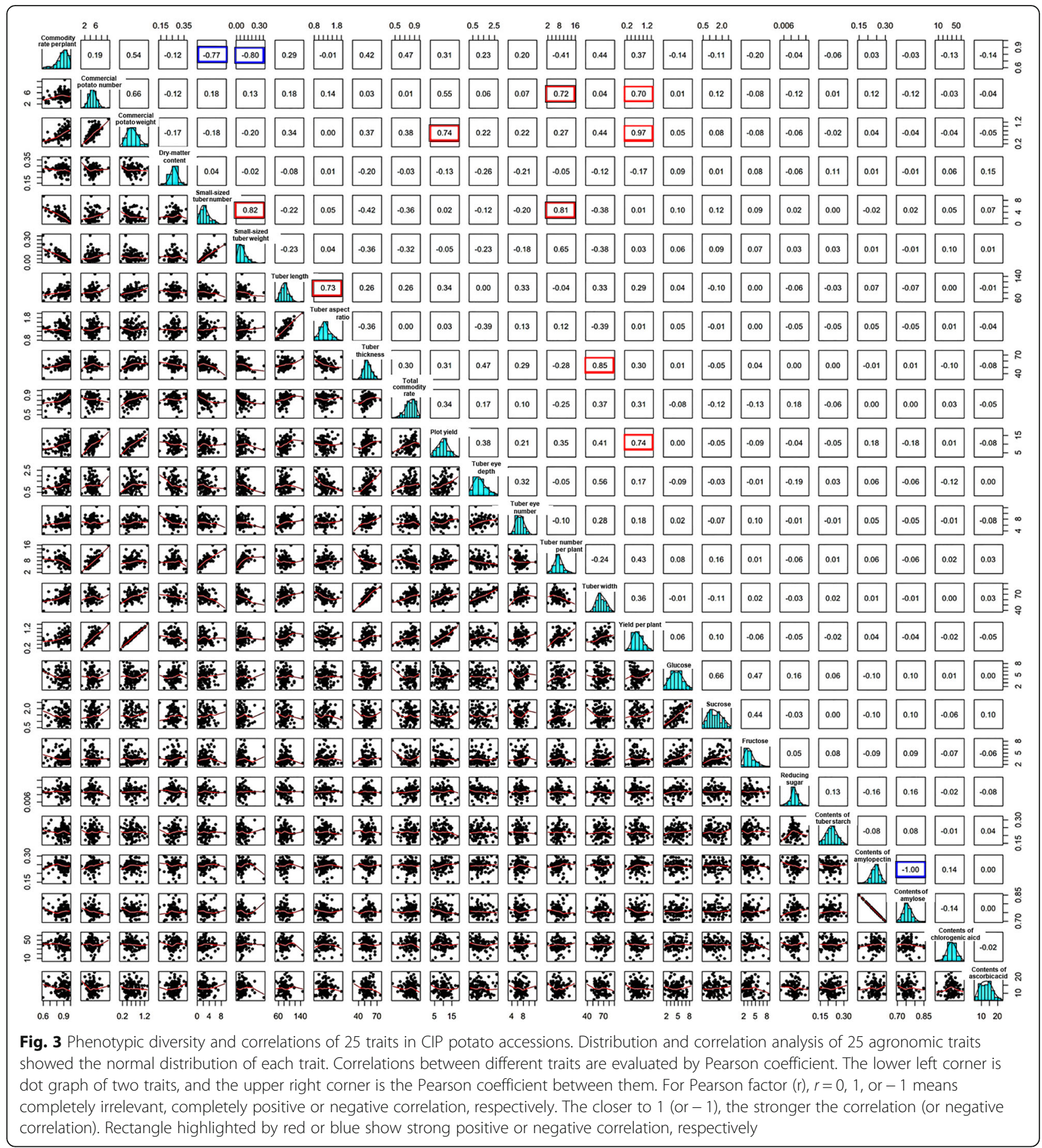

association signals containing hundreds of genes related to 15 agronomic traits were identified (Fig. 6, Fig. S2, Fig. S3, Table 1, Table S8), of which some promising loci are consistent with the previous reports.

Considering the importance of improving tuber yield and quality, tuber aspect ratio and tuber thickness are important traits for tuber shape which may affect the tuber processing and consumer preference, while increasing small-sized tuber weight is the main way to improve the tuber yield and commodity rate. Signals strongly associated with these three traits were further identified and analyzed (Fig. 6).

\section{Tuber aspect ratio}

A peak strongly associated with aspect ratio on chromosome 10 was identified within the region of previously 
A
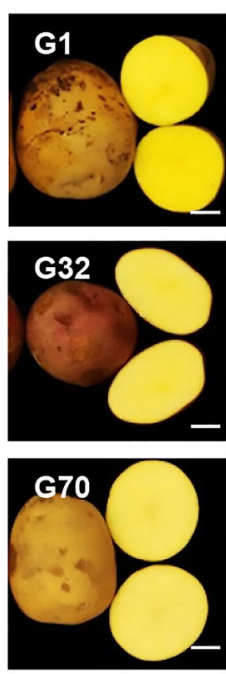

B

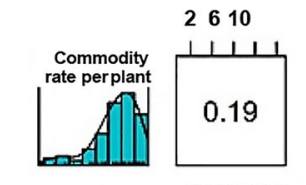

으

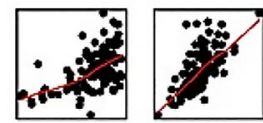

$+f$
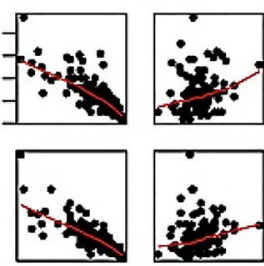

品

น
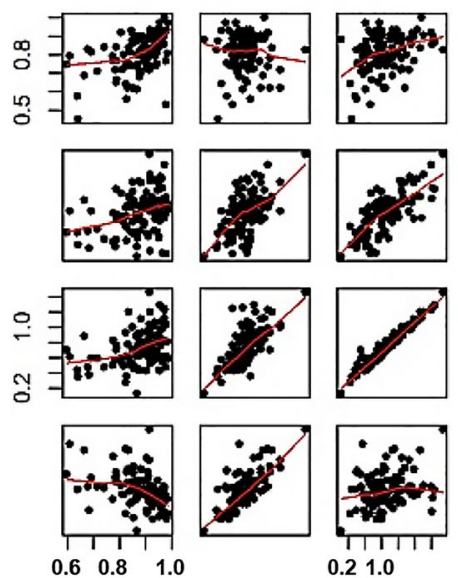

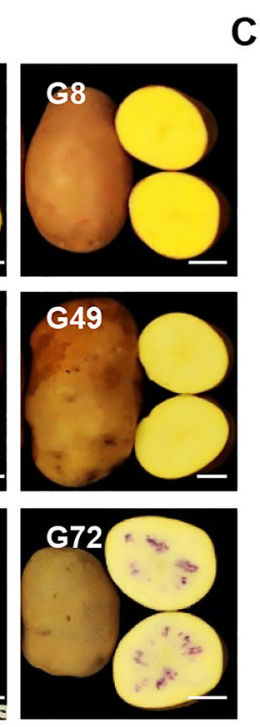

C

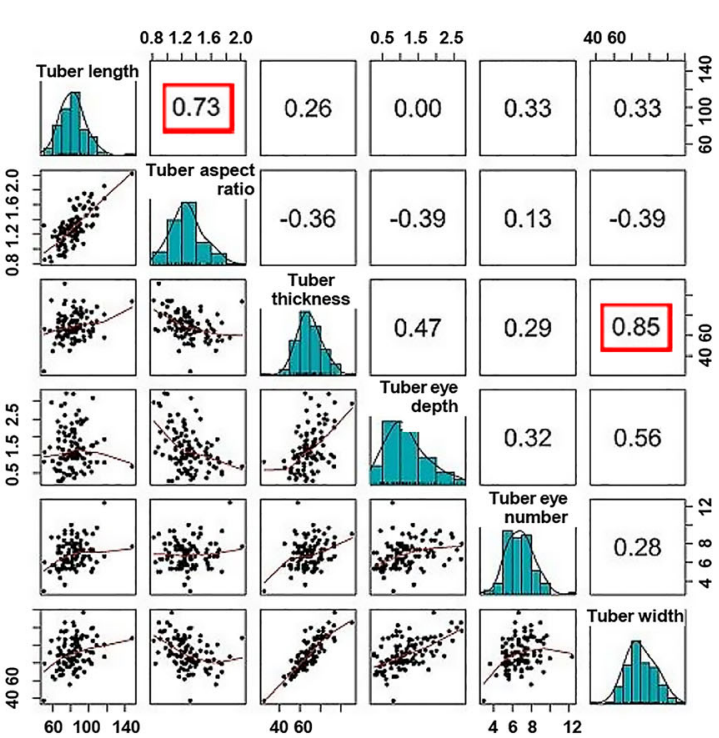

048
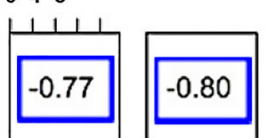

0.50 .8
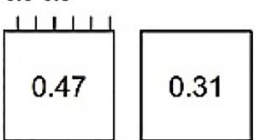

0.21 .0
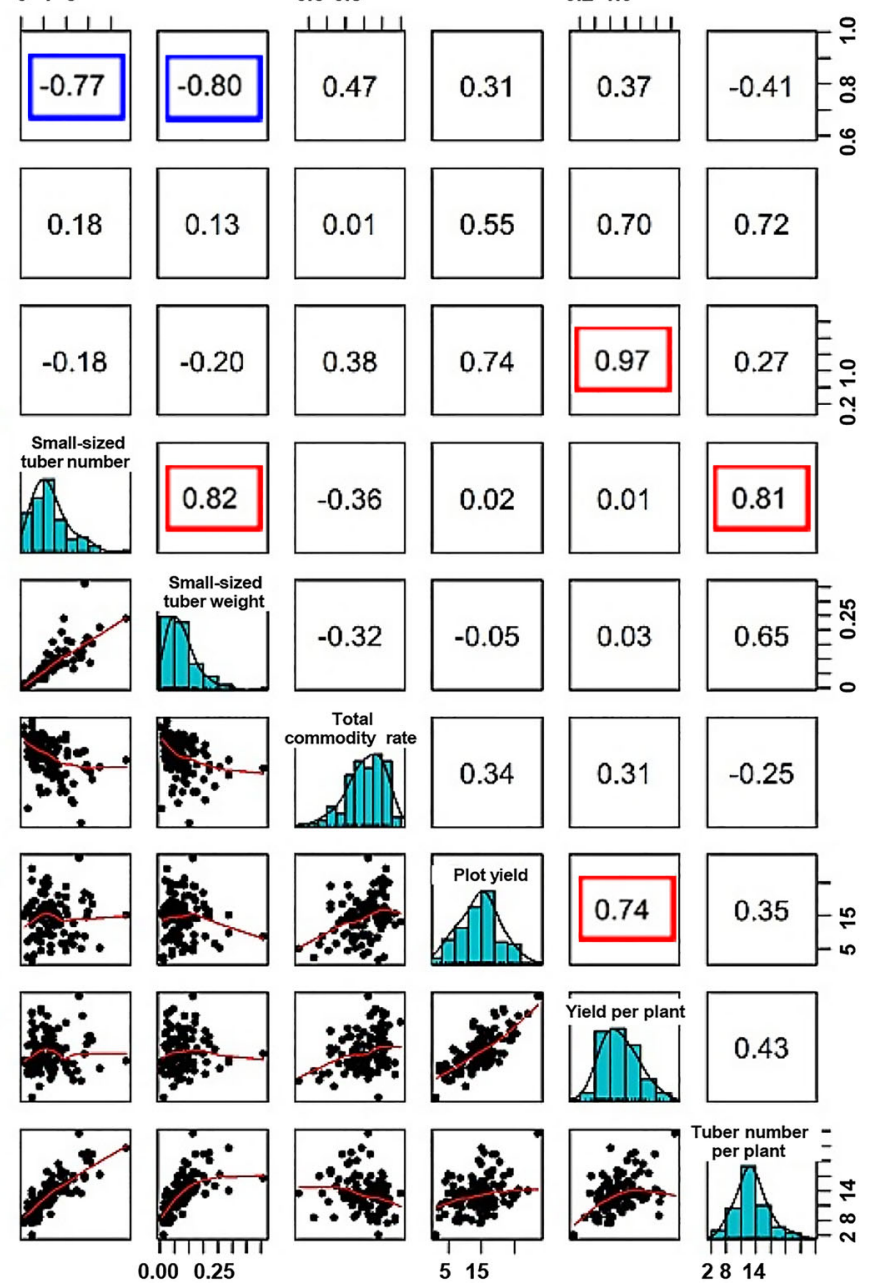

Fig. 4 (See legend on next page.) 
(See figure on previous page.)

Fig. 4 Correlations of the agronomic traits in CIP accessions. A Representative images of potato tuber with different sizes and colors, displaying the diversity of CIP accessions. Bar $=2 \mathrm{~cm}$. B Distribution and correlation analysis of yield traits showed the normal distribution of each trait and close correlations between them evaluated by Pearson factor (r). The lower left corner is the dot graph of two traits, and upper right corner is the Pearson coefficient between them. For Pearson factor ( $r$, $r=0,1$ or -1 means completely irrelevant, completely positive or negative correlation, respectively. The closer to 1 (or -1 ), the stronger the positive correlation (or negative). Rectangle highlighted by red or blue show strong positive or negative correlation, respectively. C Distribution and correlation analysis of tuber size related traits showed the normal distribution of each trait and close correlations between them evaluated by Pearson coefficient (r). Tuber size is closely related to traits including length and aspect ratio, tuber thickness and width (highlighted with red rectangle)

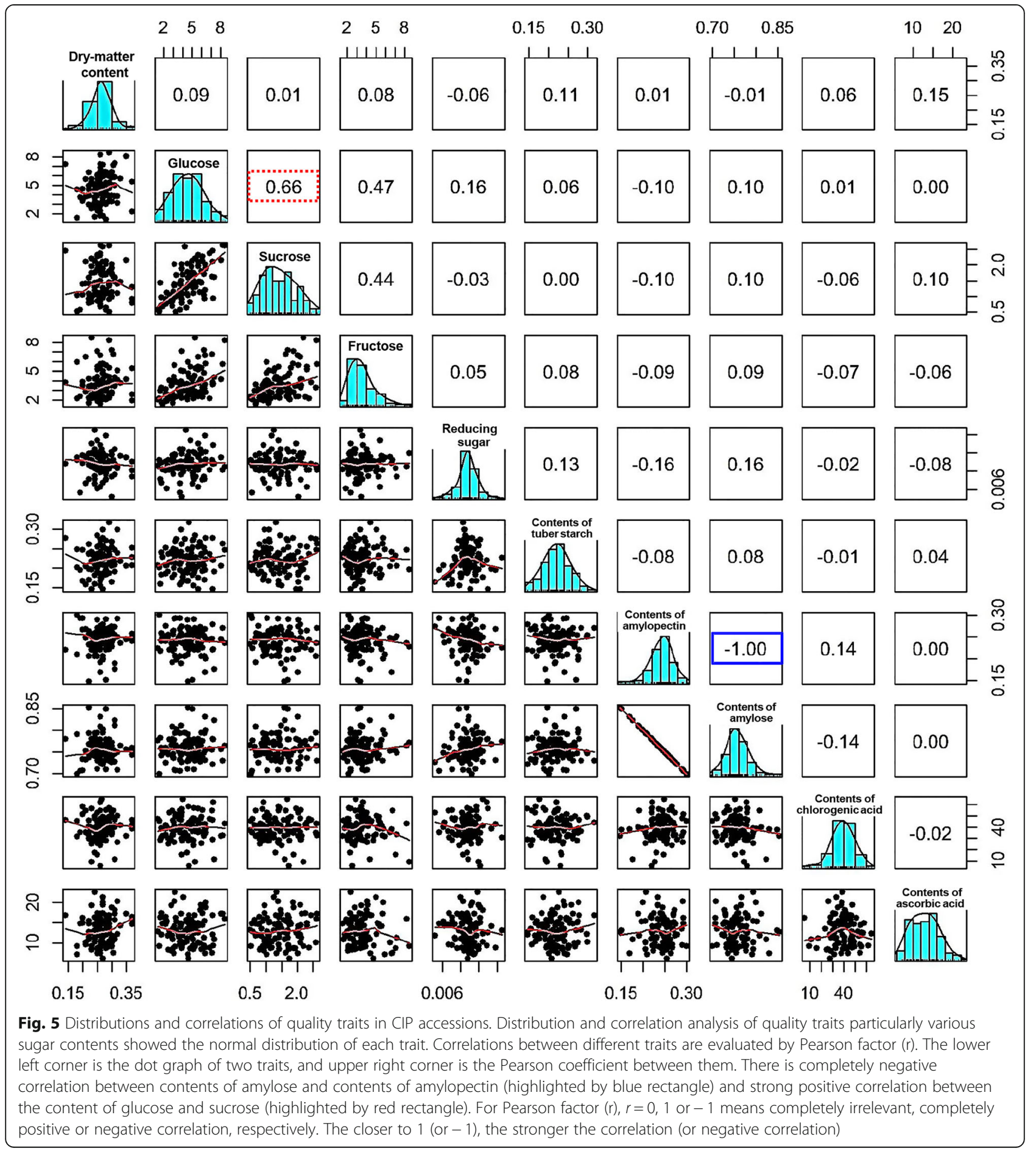




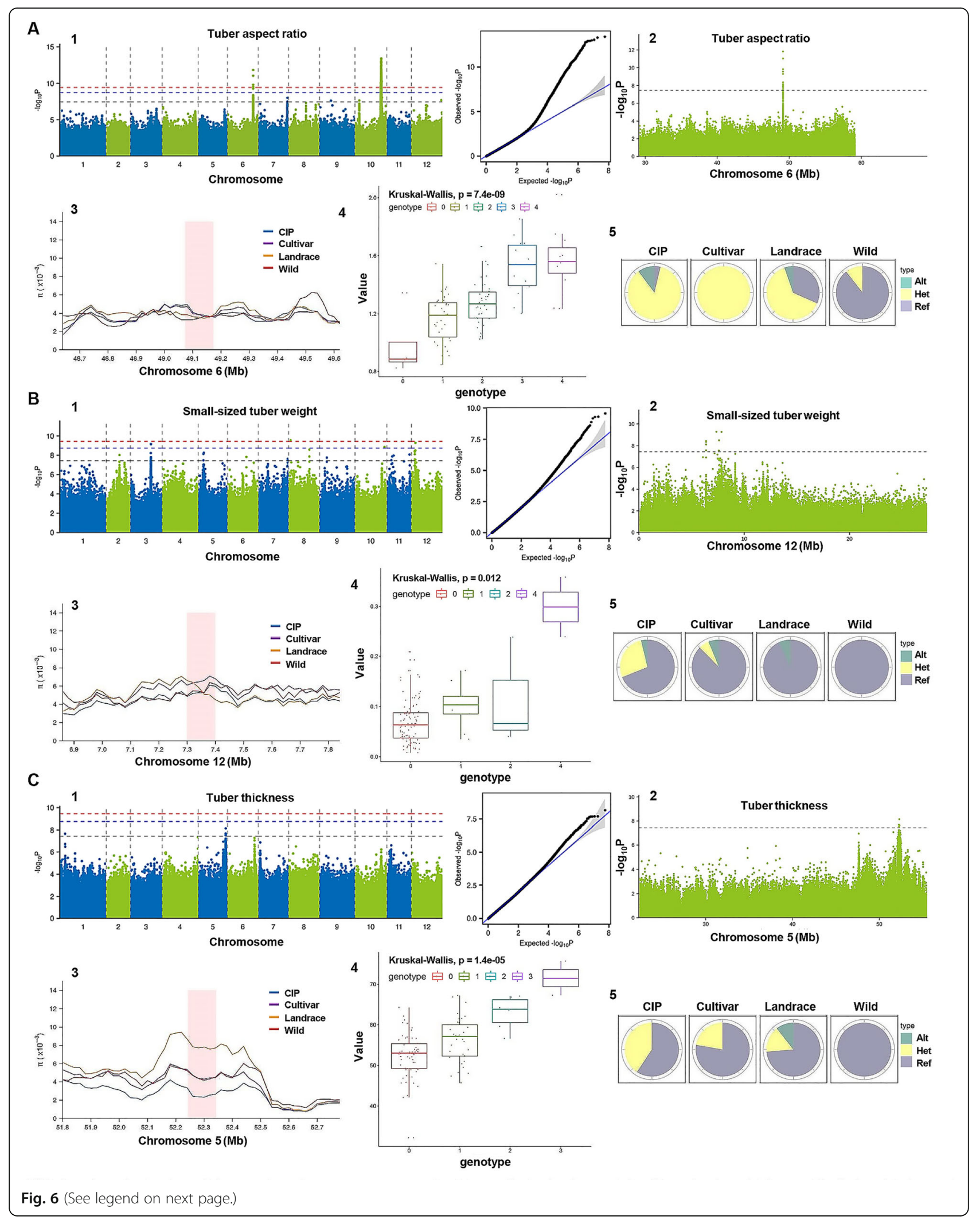




\section{(See figure on previous page.)}

Fig. 6 GWAS of potato tuber aspect ratio (A), small-sized tuber weight (B) and tuber thickness (C). 1. Manhattan plot of GWAS (left). The middle horizontal dashed line indicates the genome-wide threshold of GWAS signals with a significance level of 0.05 after Bonferroni correction [0.05/ $\left.25,591,215=1.95 \times 10^{-9}\left(-\log _{10} \mathrm{P}=8.71\right)\right]$ for multiple tests. The upper and lower horizontal dashed lines mark a significance level of 0.01 and 1 , respectively. The corresponding QQ plot (right) showed the distribution of observed $P$ values versus those expected under the null for the GWAS. 2. Candidate gene loci by enlarging the region at specific chromosome. The horizontal dashed lines indicate the genome-wide threshold of GWAS signals with a significance level of 0.05 . 3. Comparison by the $\log _{10}$ ratio of nucleotide diversity (П) revealed the nucleotide polymorphisms between Wild and CIP populations. 4. SNPs at 3'UTR and its distribution of 108 CIP accessions showed that three examined traits increased significantly with the increase of alternate allele. Numbers of various genotypes were indicated. There are five genotypes in autotetraploid $(0$, AAAA; 4, aaaa; 1, 2, 3, heterozygotic allele) and three genotypes in diploid (, AA; 1 , Aa; 2, aa). Genotype of reference allele is 0 and alternate allele is 4 (autotetraploid) or 2 (diploid). 5. Genotype distribution of key SNPs in 170 accessions revealed that Wild was dominated by Ref alleles, and Landrace and Cultivar were dominated by Het and Alt genotypes in tuber aspect ratio (A); Wild, Landrace and Cultivar was dominated by Ref alleles in small-sized tuber weight (B); Wild was dominated by Ref alleles, and Landrace and Cultivar were dominated by Ref and Het genotypes in tuber thickness (C). The four pie charts represent the proportion of different genotypes. Genotypes are indicated above (see 4) and reference allele (Ref) is 0 , alternate allele (Alt) is 4 (autotetraploid) or 2 (diploid), and heterozygotic allele (Het, 1-3 in autotetraploid or 1 in diploid)

reported Ro (Lozano et al. 2012), a main QTL controlling tuber shape. Nonetheless, there is a lack of Ro gene in the haploid reference genome used in this study (Pham et al. 2020) and the appearance of this peak region may due to the copy numbers of Ro or adjacent genes. A large peroxidase family on chromosome 10 (from Soltu.DM.10G018980 to Soltu.DM.10G019080) which closely relates to aspect ratio of tuber is dissected as well, which is consistent with that peroxidases play important roles in cell wall elongation as well as in determining shape of potato tubers.

In addition to chromosome 10, a significant GWAS signal on chromosome 6 was identified with many promising loci (Fig. 6, A1). We then analyzed the candidate gene/loci by enlarging this region and determined the accurate loci by population-differentiation statistic $\left(F_{S T}\right)$ in 20 -kb sliding windows across chromosome 6 (position 48,623,340, Fig. 6, A2). Nucleotide polymorphisms analysis with $\log _{10}$ ratio of diversity $(\Pi)$ showed the insignificant population difference between Wild and CIP populations at this position, indicating the conserved nucleotides during domestication (Fig. 6, A3; Fig. S4A).

Further analysis of SNPs on 3'-UTR and its distribution in 108 CIP populations revealed a significantly increased value of phenotypes with the increase of alternate allele (Fig. 6, A4), suggesting a close correlation between the region and phenotype. Estimation of the genotypic distribution of key SNPs in 170 samples and statistical analysis with Reference Allele (Ref), Alternate allele (Alt), and Heterozygotic Allele (Het) showed that the dominant genotype between Wild and CIP is totally differed (Fig. 6, A5), indicating that key loci in the region might be under selection and domestication.

We next searched the candidate genes mainly based on the report of homologous genes in Arabidopsis. Soltu.DM.06G023290 encodes gibberellin 20 oxidase 1 (GA20ox1) and affects tuberization and tuber growth by altering GA content, which has been validated in transgenic potato plants (Roumeliotis et al. 2013).
Interestingly, GAox family members were also under selection, revealed by the selective analysis. Soltu.DM.06G022250 encodes protein PHYTOCHROMEDEPENDENT LATE-FLOWERING (PHL) that interacts with PHYTOCHROME B (PHYB) and CONSTANS in Arabidopsis (Endo et al. 2013) and may play an role in the long day (LD)-mediated suppression of tuberization and tuber aspect ratio regulation by forming a heterodimer with PHYB (Song et al. 2019). In addition, genes involving in hormone (brassinosteroid, abscisic acid and ethylene) function are also identified, indicated the involvement of hormones in tuber shape regulation (Table S8).

\section{Small-sized tuber weight and tuber thickness}

Regions strongly associated with small-sized tuber weight were identified on chromosome 12 (Fig. 6, B1) and tuber thickness on chromosome 5 (Fig. 6, C1) respectively, i.e. chromosome 12 (position 6,848,884, Fig. 6, B2) and chromosome 5 (position 51,793,044, Fig. 6, C2) by enlarging the candidate region. Populationdifferentiation statistic $\left(F_{S T}\right)$ and nucleotide polymorphisms analysis with $\log _{10}$ ratio of diversity $(\Pi)$ showed that there is significant population difference between Wild and CIP populations in chromosome 5 associated with tuber thickness and the Wild species signals are significantly higher in this region, indicating a very strong population differentiation relating to domestication (Fig. 6, C3; Fig. S4C), while there is no significant difference in chromosome 12 associated with small-sized tuber weight, suggesting the nucleotide conservation of the loci (Fig. 6, B3; Fig. S4B). Nonetheless, value of both phenotypes increased markedly with the increase of alternate allele, indicating a tight correlation between the loci and phenotypes (Fig. 6, B4; Fig. 6, C4). Further genotypic distribution estimation of the key SNPs showed nearly half dominant genotype of CIP differed with Wild (Fig. 6, B5; Fig. 6, C5), suggesting these two loci might be under selection during domestication. 
Table 1 The 15 agronomic traits related to yield traits, quality traits and tuber appearance. Candidate loci with association signals were identified by GWAS analysis. Model, the analysis model created with GWASpoly; Chr, the chromosome on which the genes are located; Position, the physical position on chromosome, $P$-value representing the significant difference $\left(-\log _{10} P\right)$

\begin{tabular}{|c|c|c|c|c|}
\hline Trait & Model & $\mathrm{Chr}$ & Position & $P$-value \\
\hline \multirow[t]{2}{*}{ Commercial potato number } & additive & chr01 & $53,546,671$ & 7.751759221 \\
\hline & additive & chr08 & $9,289,382$ & 7.761336087 \\
\hline Commercial potato weight & additive & chr06 & $39,598,719$ & 7.635390056 \\
\hline \multirow[t]{4}{*}{ Commodity rate per plant } & additive & chr01 & $1,441,579$ & 8.379762405 \\
\hline & additive & chr05 & $8,029,132$ & 7.826109398 \\
\hline & additive & chr05 & $10,378,690$ & 7.722277807 \\
\hline & additive & chr07 & $20,336,146$ & 7.640000989 \\
\hline \multirow[t]{2}{*}{ Contents of amylopectin } & additive & chr01 & $76,598,419$ & 7.454226593 \\
\hline & additive & chr09 & $39,683,726$ & 8.201341041 \\
\hline \multirow[t]{2}{*}{ Contents of amylose } & additive & chr01 & $76,598,419$ & 7.454226593 \\
\hline & additive & chr09 & $39,683,726$ & 8.201341041 \\
\hline \multirow[t]{3}{*}{ Contents of fructose } & additive & chr01 & $1,671,891$ & 11.2415703 \\
\hline & additive & chr04 & $68,545,453$ & 7.496898574 \\
\hline & additive & chr06 & $51,907,734$ & 7.494746998 \\
\hline Contents of reducing sugar & additive & chr04 & $5,121,449$ & 8.068176837 \\
\hline \multirow[t]{6}{*}{ Small-sized tuber number } & additive & chr01 & $46,660,804$ & 8.363685848 \\
\hline & additive & chr08 & $18,192,967$ & 8.375380861 \\
\hline & additive & chr09 & $38,612,402$ & 7.756347052 \\
\hline & additive & chr09 & $65,917,552$ & 7.976222839 \\
\hline & additive & chr12 & $2,113,621$ & 8.18878601 \\
\hline & additive & chr12 & $11,738,981$ & 7.646727896 \\
\hline \multirow[t]{15}{*}{ Small-sized tuber weight } & additive & chr02 & $25,319,961$ & 8.039384488 \\
\hline & additive & chr03 & $39,235,998$ & 9.17556828 \\
\hline & additive & chr04 & $4,433,795$ & 7.449181657 \\
\hline & additive & chr05 & $10,344,300$ & 8.275191974 \\
\hline & additive & chr06 & $36,098,504$ & 7.84763694 \\
\hline & additive & chr07 & $56,127,633$ & 7.647772326 \\
\hline & additive & chr08 & $3,879,313$ & 9.571811502 \\
\hline & additive & chr08 & $39,697,219$ & 8.602640471 \\
\hline & additive & chr09 & $13,855,548$ & 7.767820029 \\
\hline & additive & chr10 & $56,486,553$ & 8.87584262 \\
\hline & additive & chr11 & $8,420,908$ & 7.506651967 \\
\hline & additive & chr11 & $10,077,049$ & 7.897851041 \\
\hline & additive & chr11 & $12,782,672$ & 7.98400409 \\
\hline & additive & chr11 & $43,116,815$ & 8.094322885 \\
\hline & additive & chr12 & $7,348,885$ & 9.309574598 \\
\hline Total commodity rate & additive & chr07 & $7,063,854$ & 7.666711254 \\
\hline \multirow[t]{5}{*}{ Tuber aspect ratio } & additive & chr06 & $49,123,341$ & 11.83625679 \\
\hline & additive & chr07 & $55,669,850$ & 8.023519864 \\
\hline & additive & chr09 & $21,823,977$ & 7.632673794 \\
\hline & additive & chr10 & $8,238,500$ & 7.657560212 \\
\hline & additive & chr10 & $50,375,842$ & 13.41468486 \\
\hline
\end{tabular}


Table 1 The 15 agronomic traits related to yield traits, quality traits and tuber appearance. Candidate loci with association signals were identified by GWAS analysis. Model, the analysis model created with GWASpoly; Chr, the chromosome on which the genes are located; Position, the physical position on chromosome, $P$-value representing the significant difference $\left(-\log _{10} P\right)\left(C_{0}\right.$ tinued)

\begin{tabular}{lllll}
\hline Trait & Model & Chr & Position & P-value \\
\hline & additive & chr12 & $57,239,887$ & 7.691485609 \\
Tuber eye depth & additive & chr10 & $49,591,276$ & 9.441733516 \\
Tuber length & additive & chr09 & $25,598,280$ & 7.486629211 \\
& additive & chr09 & $45,761,555$ & 7.474995731 \\
& additive & chr10 & $50,067,847$ & 7.770368725 \\
Tuber number per plant & additive & chr08 & $8,407,989$ & 7.809295303 \\
Tuber thickness & additive & chr01 & $9,983,088$ & 7.677787051 \\
& additive & chr05 & $52,293,045$ & 8.159705715 \\
\hline
\end{tabular}

At the candidate region of chromosome 12, AtOSM34 regulates pathogen response and cell cycle in Arabidopsis (Sozzani et al. 2008) and whose homolog in potato was identified from Soltu.DM.12G007830 to Soltu.DM.12G007890, which may affect the small-sized tuber weight by regulating cell division. In addition, Soltu.DM.12G008030 and Soltu.DM.12G008040 encode SMALL AUXIN UPREGULATED 66 (SAUR66, Lin et al. 2005) and SAUR20 (Spartz et al. 2012) proteins that involve in auxin effects and regulate sucrose efflux in Arabidopsis. Gene Soltu.DM.12G008510 encodes sugar transporter SWEET7 and involves in sucrose efflux and phloem-mobile tuberization signals ( $\mathrm{Qu}$ et al. 2012). These genes regulate tuberization as well as tuber growth and development, and may finally affect smallsized tuber weight and yield.

Many hormone related genes, particularly those involving in GA signaling and effects, including Soltu.DM.05G023410 (DELLA protein RGA-LIKE 2, RGL2, Gómez et al. 2019) and Soltu.DM.05G023320 (gibberellin 2-oxidase 6) as well as homolog of PHYB Soltu.DM.05G023390 (Jiang et al. 2020) were selected and identified with association with tuber thickness on chromosome 5.

In addition, key loci associated with other agronomic traits which are closely correlated with potato appearance, yield and quality, and crucial for its commodity value are identified (Fig. S2, Fig. S3, Table 1, Table S8). All associated genes were validated with the expression pattern at different stages of tuber development (Fig. S5) by using tuber transcriptome data (http://solanaceae. plantbiology.msu.edu/pgsc_download.shtml). The identified candidate regions and genes associated with key agronomic traits may be important targets for biotechnological or breeding approaches in the future.

\section{Discussions}

Vegetative propagated crops such as potato are globally important in terms of agricultural production, long-term history of early agriculture and plant domestication as well as planning for more sustainable agricultural futures (Harris, 1972). Domestication represents humanmediated plant evolution revealing a combination of permanent genetic changes and impermanent plastic responses to practices of cultivation or growth environment (Denham et al. 2020). There is still a lack of excellent cultivated varieties with higher yield, better quality and stronger adaptability especially under biotic and abiotic stresses. In addition, the biggest obstacle for molecular research and breeding is the lack of genomic information and identification of gene function due to the analysis difficulty and complexity of autotetraploid genomes. We here reported the resequencing of 108 autotetraploid CIP accessions by whole-genome sequencing and dissected 138 high-confidence selective sweeps comprising 54 predicted genes with selective analysis. In addition, we established and improved the autotetraploid analysis system with GWASpoly software and identified 50 candidate loci associating with 15 agronomic traits related to tuber yield and quality, which provides valuable genomic resources for autotetraploid potato cultivars and lays a good foundation for autotetraploid potato research and further molecular breeding. Identified correlations of diverse agronomic traits not only lay the foundation for elucidating the character elements of potato varieties constitute, properties and the relationship between trait components and total traits, but also help to clarify the process of selection and domestication of common cultivars as well as the GWAS analysis, so as to guide the selection of varieties and potato breeding.

Revelation of genomic sequence at the whole-genome level and identification of functional genes is urgent and essential considering the importance of autotetraploid potato cultivars in product and breeding. Resequencing of autotetraploid CIP accessions with whole-genome sequencing (WGS) fills in blanks of genomic information for autotetraploid cultivars and referenced the newly revealed haploid genome which is a chromosome-scale 
long-read assembled reference genome (DM v6.1, 16) due to the lack of autotetraploid potato reference genome. Analysis of the diverse agronomic traits is also essential since there are significant differences in yield, resistance and quality, genetic background, ecological adaptability, outward appearance and flavor types. Additionally, 138 high-confidence selective sweeps comprising 54 predicted genes related to flowering time, temperature sensitivity and cell division were also identified by selective interval analysis, which will help to understand the variable traits and complex domestication process of potato adaptation to changed environment and are potential new molecular markers that can be applied for potato breeding. Moreover, the SNPs in candidate genes of GWAS are related to domestication, indicating the changes of key genes in potato selection. Genome variation correlated with domestication revealed how genome reshaped under selection for better phenotypic traits and provides a rich resource for potato cultivation and breeding.

Complex genome with different ploidy of potato and the quantitative nature of most agronomic traits are challenging for accurately understanding the phenotype and genotype relations. GWAS might not be as promising in polyploid species as in diploids since the software tailored to polyploidy is lacking although it has been widely used in diploid species to study the complex traits in diversity and breeding populations. Appropriate GWAS software tailored to polyploidy is the main constraint and biggest challenge to autotetraploid potato species. In this study, we introduced GWASpoly in R, a software tailored for polyploidy and has been utilized in sugarcane and chrysanthemum (Yang et al. 2020; Sumitomo et al. 2019), which could model different types of polyploid gene actions in GWAS, including additive, simplex dominant, and duplex dominant (Rosyara et al. 2016). Combination of GWASpoly and WGS provide more accurate analysis of polyploid genome and may shed light on autotetraploid analysis of other species.

Considering the research combining GWAS with WGS is relative rare in potatoes especially in autotetraploid potato, identification of 50 promising candidate loci including hundreds of genes associating with 15 important agronomic traits of autotetraploid potato by GWAS analysis not only provides new markers for molecular research but also contributes the elucidation of gene effects and functional mechanisms. Although there is still need to further verify the identified loci and genes (gene editing approach will help), identification of them will provide informative clues for potato genetics and breeding practice.

\section{Methods}

\section{Plant materials and growth condition}

A total of 108 potato cultivars in the high generation line catalog of International Potato Center (CIP, https:// cipotato.org/catalogue) were analyzed (Supplemental Table 1). These cultivars contain 11 populations from 11 hybridized combinations. The male and female parents of each combination have complex genetic diversity, including Varieties, Population A, BW (bacterial blight resistance), Cycle 0, Cycle 1, Others, B3C1, LBHT-1 (late maturity, late blight resistance, high starch, suitable for processing Fried, heat-resisting), Intermediate LT-LB (medium-early maturity, dishes, late blight resistance), LTVR/LD (adapt to the long day) and LTVR (heat, virus, vegetable, low altitude high temperature adaptability).

Pilot planting was carried out in Weiyuan city, Gansu province in 2016 and 2017. Briefly, a random block design was adopted to sow $50 \mathrm{~g}$ cutted potato tuber and each variety has 3 plots whose area was $1.1 \mathrm{~m} \times 2.5 \mathrm{~m}$ by single ridge and double row planting with $40 \mathrm{~cm}$ row spacing and $25 \mathrm{~cm}$ plant spacing. No fertilization and no irrigation were used in all experiments and $10-15 \mathrm{~cm}$ depth of soil was covered for seedling consolidation. Phenotypic observation and measurement of various traits were analyzed and evaluated every year.

\section{Phenotyping}

For yield traits, total commodity rate, commodity rate per plant, commercial potato number, commercial potato weight, small-sized tuber number, small-sized tuber weight, tuber number per plant, yield per plant and plot yield were analyzed according to previous study (Zhang and Tian, 2007). For external properties, tuber length (L) was measured from top of tuber to the umbilical cord lengthwise with vernier caliper after washing and drying the tuber. Tuber width (W) was measured horizontally from the widest part of tuber and thickness was measured from the tabletop to the highest point of tuber. Tuber aspect ratio $=\mathrm{L} / \mathrm{W}$. The depth of tuber's eye was measured with a depth ruler, and average number and depth of buds were calculated.

For quality traits, dry matter content was measured by drying weighing method, content of tuber starch was determined by specific gravity method, and contents of amylose and amylopectin by double wavelength method (Zhang and Tian, 2007). Contents of sugar including sucrose, fructose, glucose and reducing sugar, and acid including ascorbic acid, chlorogenic acid were determined according to previously reported method (Ohara-Takada et al. 2005).

\section{DNA extraction and sequencing}

Total genomic DNA was extracted from tuber tissues using the DNeasy Plant Mini Kit (Qiagen). All DNA samples were diluted to $20 \mathrm{ng} \cdot \mu^{-1}$ concentration and stored at $-20^{\circ} \mathrm{C}$. For each accession of CIP panel, at least $5 \mu \mathrm{g}$ of DNA was used to construct a sequencing library with an Illumina TruSeq DNA Sample Prep Kit 
according to the manufacturer's instructions. Paired-end sequencing (150 base pairs) of each library was performed on an Illumina HiSeq X Ten system at Shanghai OEbiotech Co Ltd. (Shanghai, China).

\section{Reads mapping and variants calling}

For the reported 67 accession panel, resequencing data for cultivars, landraces and wild were downloaded from the NCBI database under PRJNA378971 (Hardigan et al. 2017). The raw paired-end reads of 170 accessions were trimmed to remove the adaptors and low-quality bases using FastP (Campos et al. 2006). Reads were filtered with a sliding window of size 4 , with average Phred score scale of 20 within the window. Trimmed reads were mapped to the $S$. tuberosum group Phureja DM reference genome (Pham et al. 2020) (Ensembl release 42) using bwamem (Li and Durbin, 2009) (version: 0.7-17) with default parameters. After alignment, Picard tools (version: 2.18.17, http://broadinstitute.github.io/picard/) were used to remove the PCR duplicates according to the mapping coordinates.

Detection of variation is performed following the best practice workflow recommended by GATK (18) (version 3.8.1). In brief, the variants were called for each accession by GATK Haplotype Caller. A joint genotyping step for comprehensive variations union was performed on the gVCF, the ploidy was set 2 or 4 according to the ploidy of accessions. In the filtering step, the SNP filter expression was set as QD $<2.0 \| \mathrm{MQ}<20.0$ || FS $>60.0$ || SOR > 3.0 || MQRankSum <-12.5 || ReadPosRankSum $<-8.0$, and the InDel filter expression was set as $\mathrm{QD}<2.0 \quad\|\mathrm{FS}>200.0 \quad\| \quad \mathrm{MQ}<40.0 \quad\|\quad \mathrm{SOR}>10.0 \quad\|$ ReadPosRankSum $<-20.0$.

Three subsets of potato SNPs were defined using following filtering criteria: (1) the basic set of 27,565,997 SNPs were created excluding non bi-allelic, $>20 \%$ missing calls and MAF < 5\%; (2) Phylogeny SNP set of 72,923 SNPs randomly sampled at equal counts from genomewide 10-kb windows; (3) GWAS SNP set of 27,494,422 SNPs were created by selecting 108 phenotyped CIP individuals by filtering $\mathrm{MQ}<40$ and $\mathrm{MAF}<0.05$ form all 170 samples raw vcf.

SNPs and InDels annotation were performed according to the wild castor genome using SNPeff (Cingolan et al. 2012) (version: $4.3 \mathrm{~T}$ ). The coverage of each accession against each chromosome of grapevine genome was counted based on aligned BAM file using QualiMap (García-Alcalde et al. 2012) (version: 2.2.1) software.

\section{Population genetics analysis}

A phylogenetic tree was constructed from the phylogeny SNP data by using neighbor-joining method in program PHYLIP (Felsenstein, 1989) (version: 3.697, http://evolution.genetics.washington.edu/phylip.html) and colored by $\mathrm{R}$ package ggtree (Yu et al. 2017), and IBS distance matrices were calculated using PLINK (Purcell et al. 2007) (version: 1.9). Principal components analysis (PCA) (Price et al. 2006) was performed with EIGENSOFT software. Population structure was analyzed using the ADMIXTURE (Alexander et al. 2009) (version: 1.3) program with a block-relaxation algorithm by collapsed heterozygotes in the dosage model to $0 / 1$ genotypes.

To explore the convergence of individuals, we predefined the number of genetic clusters $\mathrm{K}$ from 2 to 10 and ran the cross-validation error $(\mathrm{CV})$ procedure. ADMIXTURE was then run again on the whole core SNP set 10 times with varying random seeds; the Q-matrices were aligned using pong (Behr et al. 2016) software and clustered on the basis of similarity. The matrices belonging to the largest cluster were averaged to produce the final matrix of admixture proportions.

\section{Genome scanning for selective sweep signals}

A genetic differentiation $\left(F_{S T}\right)$, nucleotide diversity $\left(\theta_{\pi}\right)$ and XP-CLR analysis based cross approach was performed to investigate the selection signals across the whole genome. A 20-kb sliding window with 10-kb step approach was applied to quantify the $F_{S T}, \theta_{\pi}$ and XP-CLR by using $\mathrm{R}$ package PopGenome (Pfeifer et al. 2014). The candidates that meet both top $5 \%$ of the $F_{S T}$ and $\theta_{\pi}$, and $1 \%$ of the XP-CLR were regarded as selective signals.

\section{Correlation analysis between agronomic traits of CIP accessions}

Non-linear regression analysis was performed using Rpsych software and presented with Pearson factor. Pearson coefficients were calculated with $\mathrm{lm}=$ True (linear regression fits are shown for both $\mathrm{y}$ by $\mathrm{x}$ and $\mathrm{x}$ by $\mathrm{y}$. Correlation ellipses are also shown.)

\section{Genome-wide association analysis}

Since the DAPC analysis showed the best cluster of 108 CIP accessions were 2, we only considered cryptic kinship relationships (K) to minimize false positives and increase statistical power. GWASpoly (Rosyara et al. 2014) is tailored for autopolyploid based on the $\mathrm{Q}+\mathrm{K}$ mixed model and could model gene actions for polyploids. Six models were used for GWAS by using GWASpoly, including general model, additive model, two simplex dominant models (1-dom-ref and 1-dom-alt), diplogeneral model, and diplo-additive model. We defined the whole-genome significance cutoff with the adjusted Bonferroni test threshold, which was set as $P<0.05$ / $27,494,422=1.82 \times 10^{-9}\left(-\log _{10} \mathrm{P}=8.74\right)$. 


\section{Transcriptome analysis}

FPKM values of all representative transcripts across 40 $\mathrm{DM}$ and $16 \mathrm{RH}$ libraries were downloaded from the PGSC data (http://solanaceae.plantbiology.msu.edu/ pgsc_download.shtml). Genes in the upstream and downstream 500-kb region of the leading SNPs of each trait were selected and expression pattern associated with the trait was shown except genes whose FPKM equals to 0 across all libraries.

\section{Abbreviations}

GWAS: genome-wide association study; NGS: next-generation sequencing; WGS: whole-genome sequencing; SSR: simple sequence repeat; SNP: Single Nucleotide Polymorphisms; InDel: Insertion and Deletion; BIC: Bayesian Information Criterion; PCA: principal-component analysis

\section{Supplementary Information}

The online version contains supplementary material available at https://doi. org/10.1186/s43897-022-00027-y.

Additional file 1: Fig. S1. Cross validation and Bayesian Information Criterion analysis identified the optimal number of subpopulations. Fig. S2. Manhattan plot by GWAS for the 12 agronomic traits with association signals. Fig. S3. Manhattan plot by GWAS for 10 agronomic traits with weak association signals. Fig. S4. Pairwise fixation index $\left(F_{S T}\right)$ in potato tuber aspect ratio, small-sized tuber weight and tuber thickness. Fig. S5. Transcriptome analysis of the candidate genes by GWAS.

Additional file 2: Supplemental Table S1. Sample information for 108 CIP accessions

Additional file 3: Supplemental Table S2. Overview of sequencing of samples.

Additional file 4: Supplemental Table S3. Statistics of SNPS and InDels for CIP 108 accessions.

Additional file 5: Supplemental Table S4. Statistics of SNPS and InDels for 170 accessions.

Additional file 6: Supplemental Table S5. Selected intervals and genes between Wild and CIP cultivars.

Additional file 7: Supplemental Table S6. Selected intervals and genes between reported and CIP cultivars.

Additional file 8: Supplemental Table S7. Phenotypic information of 108 CIP accessions.

Additional file 9: Supplemental Table S8. GWAS signals and candidate genes.

\section{Acknowledgments}

This work was supported by Ten Thousand Talent Program. We thank Mr Zhi-Gui Bao (OEbiotech) for valuable suggestions for data analysis.

\section{Authors' contributions}

Z.H.X. and H.W.X. designed the research; F.Z., L.Q. and Y.C.G performed experiments, sequencing and data analysis; L.Q. drafted and H.W.X. wrote the paper. All authors read and approved the final manuscript.

\section{Funding}

This study was supported by Ten Thousand Talent Program.

\section{Availability of data and materials}

All relevant data are within the manuscript and Supporting information files. Resequencing data of 108 CIP accessions can be found in NCBI database under SUB9867284.

\section{Declarations}

Ethics approval and consent to participate

Not applicable.

\section{Consent for publication}

Not applicable.

\section{Competing interests}

The authors declare that they have no competing interests.

\section{Author details}

${ }^{1}$ College of Agronomy, Gansu Provincial Key Laboratory of Aridland Crop Science, Gansu Key Laboratory of Crop Improvement \& Germplasm Enhancement, Gansu Agricultural University, Lanzhou 730070, China. ${ }^{2}$ Shanghai Collaborative Innovation Center of Agri-Seeds, Joint Center for Single Cell Biology, School of Agriculture and Biology, Shanghai Jiao Tong University, Shanghai 200240, China. ${ }^{3}$ Shanghai OEbiotech, Shanghai 201210, China. ${ }^{4}$ National Key Laboratory of Plant Molecular Genetics, CAS Center for Excellence in Molecular Plant Sciences, Chinese Academy of Sciences, Shanghai 200032, China.

Received: 15 November 2021 Accepted: 1 February 2022

Published online: 10 February 2022

\section{References}

Alexander DH, Novembre J, Lange K. Fast model-based estimation of ancestry in unrelated individuals. Genome Res. 2009;19(9):1655-64. https://doi.org/10.11 01/gr.094052.109.

Balmer Y, Vensel WH, Tanaka CK, Hurkman WJ, Gelhaye E, Rouhier N, et al. Thioredoxin links redox to the regulation of fundamental processes of plant mitochondria. Proc Natl Acad Sci U S A. 2004;101(8):2642-7. https://doi.org/1 0.1073/pnas.0308583101.

Behr AA, Liu KZ, Liu-Fang G, Nakka P, Ramachandran S. Pong: fast analysis and visualization of latent clusters in population genetic data. Bioinformatics. 2016;32(18):2817-23. https://doi.org/10.1093/bioinformatics/btw327.

Birch PRJ, Bryan G, Fenton B, Gilroy EM, Hein I, Jones JT, et al. Crops that feed the world 8: potato: are the trends of increased global production sustainable? Food Secur. 2012;4(4):477-508. https://doi.org/10.1007/s12571-012-0220-1.

Campos D, Noratto G, Chirinos R, Arbizu C, Roca W, Cisneros-Zevallos L. Antioxidant capacity and secondary metabolites in four species of Andean tuber crops: native potato (Solanum sp.), mashua (Tropaeolum tuberosum Ruiz \& Pavon), Oca (Oxalis tuberosa Molina) and ulluco (Ullucus tuberosus Caldas). J Sci Food Agric. 2006;86(10):1481-8. https://doi.org/10.1002/jsfa.2529.

Charng YY, Liu HC, Liu NY, Chi WT, Wang CN, Chang SH, et al. A heat-inducible transcription factor, $\mathrm{HsfA} 2$, is required for extension of acquired thermotolerance in Arabidopsis. Plant Physiol. 2007;143(1):251-62.

Cingolan P, Platts A, Wang LL, Coon M, Nguyen T, Wang L, et al. A program for annotating and predicting the effects of single nucleotide polymorphisms, SnpEff: SNPs in the genome of Drosophila melanogaster strain w(1118); iso-2; iso-3. Fly. 2012;6(2):80-92. https://doi.org/10.4161/fly.19695.

Denham T, Barton H, Castillo C, Crowther A, Dotte-Sarout E, Florin SA, et al. The domestication syndrome in vegetatively propagated field crops. Annu Bot. 2020;125(4):581-97. https://doi.org/10.1093/aob/mcz212.

Diamond J. Evolution, consequences and future of plant and animal domestication. Nature. 2002;418(6898):700-7. https://doi.org/10.1038/na ture01019.

Dutt S, Manjul AS, Raigond P, Singh B, Siddappa S, Bhardwaj V, et al. Key players associated with tuberization in potato: potential candidates for genetic engineering. Crit Rev Biotechnol. 2017;37(7):942-57.

Endo M, Tanigawa Y, Murakami T, Araki T, Nagatani A. PHYTOCHROMEDEPENDENT LATE-FLOWERING accelerates flowering through physical interactions with phytochrome B and CONSTANS. Proc Natl Acad Sci USA. 2013;110(44):18017-22. https://doi.org/10.1073/pnas.1310631110.

Felsenstein J. PHYLIP - phylogeny inference package-v3.2. Cladistics. 1989:164-6. Fuller DQ, Allaby RG. Fruit development and seed dispersal (ed. Ostergaard, L.). Blackwell. 2009;38:238-95.

García-Alcalde F, Okonechnikov K, Carbonell J, Cruz LM, Götz S, Tarazona S, et al. Qualimap: evaluating next-generation sequencing alignment data. Bioinformatics. 2012;28(20):2678-9. https://doi.org/10.1093/bioinformatics/ bts503. 
Gómez MD, Fuster-Almunia C, Javier Ocaña-Cuesta J, Jose M, Alonso JM, PérezAmador MA. RGL2 controls flower development, ovule number and fertility in Arabidopsis. Plant Sci. 2019;281:82-92. https://doi.org/10.1016/j.plantsci.201 9.01.014.

Goulas E, Schubert M, Kieselbach T, Kleczkowski LA, Gardeström P, Schröder W, et al. The chloroplast lumen and stromal proteomes of Arabidopsis thaliana show differential sensitivity to short- and long-term exposure to low temperature. Plant J. 2006;47(5):720-34. https://doi.org/10.1111/j.1365-313X.2 006.02821.x.

Gutaker RM, Weiß CL, Ellis D, Anglin NL, Knapp S, Luis Fernández-Alonso J, et al. The origins and adaptation of European potatoes reconstructed from historical genomes. Nat Ecol Evol. 2019;3(7):1093-101. https://doi.org/10.103 8/s41559-019-0921-3.

Hardigan MA, Laimbeer FPE, Newton L, Crisovan E, Hamilton JP, Vaillancourt B, et al. Genome diversity of tuber-bearing Solanum uncovers complex evolutionary history and targets of domestication in the cultivated potato. Proc Natl Acad Sci USA. 2017;114(46):E9999-E10008. https://doi.org/10.1073/ pnas. 1714380114

Harris DR. The origins of agriculture in the tropics. Am Scientist. 1972;60:180-93.

Huang X, Kurata N, Wei X, Wang Z, Wang A, Zhao Q, et al. A map of rice genome variation reveals the origin of cultivated rice. Nature. 2012;490(7421): 497-501. https://doi.org/10.1038/nature11532.

Jiang J, Xiao Y, Chen H, Hu W, Zeng L, Ke H, et al. Retrograde induction of phyB orchestrates ethylene-auxin hierarchy to regulate growth. Plant Physiol. 2020; 183(3):1268-80. https://doi.org/10.1104/pp.20.00090.

Kuang HH, Wei FS, Marano MR, Wirtz U, Wang XX, Liu J, et al. The R1 resistance gene cluster contains three groups of independently evolving, type I R1 homologues and shows substantial structural variation among haplotypes of Solanum Demissum. Plant J. 2005;44(1):37-51. https://doi.org/10.1111/j.13 65-313X.2005.02506.X.

Levy D, Veilleux RE. Adaptation of potato to high temperatures and salinity. Am J Potato Res. 2007;84(6):487-506. https://doi.org/10.1007/BF02987885.

$\mathrm{Li} \mathrm{H}$, Durbin R. Fast and accurate short read alignment with burrows-wheeler transform. Bioinformatics. 2009;25(14):1754-60. https://doi.org/10.1093/ bioinformatics/btp324.

Li N, Lin B, Wang H, Li X, Yang F, Ding $X$, et al. Natural variation in ZmFBL41 confers banded leaf and sheath blight resistance in maize. Nat Genet. 2019; 51(10):1540-8. https://doi.org/10.1038/s41588-019-0503-y.

Li Y, Colleoni C, Zhang J, Liang Q, Hu Y, Ruess H, et al. Genomic analyses yield markers for identifying agronomically important genes in potato. Mol Plant. 2018;11(3):473-84. https://doi.org/10.1016/j.molp.2018.01.009

Liao C, Zheng Y, Guo Y. MYB30 transcription factor regulates oxidative and heat stress responses through ANNEXIN-mediated cytosolic calcium signaling in Arabidopsis. New Phytol. 2017;216(1):163-77. https://doi.org/10.1111/nph.14 679.

Lin WH, Wang Y, Mueller-Roeber B, Brearley CA, Xu ZH, Xue HW. At5PTase13 modulates cotyledon vein development through regulating auxin homeostasis. Plant Physiol. 2005;139(4):1677-91. https://doi.org/10.1104/pp.1 05.067140.

Lozano R, Ponce O, Ramirez M, Mostajo N, Orjeda G. Genome-wide identification and mapping of NBS-encoding resistance genes in Solanum tuberosum group Phureja. PLoS One. 2012;7(4):e34775. https://doi.org/10.1371/journal. pone.0034775.

Martinez-Garcia JF, Garcia-Martinez JL, Bou J, Prat S. The interaction of gibberellins and photoperiod in the control of potato tuberization. J Plant Growth Regul. 2001;20(4):377-86. https://doi.org/10.1007/s003440010036.

McKenna A, Hanna M, Banks E, Sivachenko A, Cibulskis K, Kernytsky A, et al. The genome analysis toolkit: a MapReduce framework for analyzing nextgeneration DNA sequencing data. Genome Res. 2010;20(9):1297-303. https:// doi.org/10.1101/gr.107524.110.

Ohara-Takada A, Matsuura-Endo C, Chuda Y, Ono H, Yada H, Yoshida M, et al. Change in content of sugars and free amino acids in potato tubers under short-term storage at low temperature and the effect on acrylamide level after frying. Biosci Biotechnol Biochem. 2005;69(7):1232-8. https://doi.org/1 $0.1271 / \mathrm{bbb} .69 .1232$

Pfeifer B, Wittelsburger U, Ramos-Onsins SE, Lercher MJ. PopGenome: an efficient swiss army knife for population genomic analyses in R. Mol Biol Evol. 2014; 31(7):1929-36. https://doi.org/10.1093/molbev/msu136.

Pham GM, Hamilton JP, Wood JC, Burke JT, Zhao H, Vaillancourt B. Construction of a chromosome-scale long-read reference genome assembly for potato. Gigascience. 2020;9(9):giaa100.
Price AL, Patterson NJ, Plenge RM, Weinblatt ME, Shadick NA, Reich D. Principal components analysis corrects for stratification in genome-wide association studies. Nat Genet. 2006;38(8):904-9. https://doi.org/10.1038/ng1847.

Purcell S, Neale S, Todd-Brown K, Thomas L, Ferreira MAR, Bender D, et al. PLINK: a tool set for whole-genome association and population-based linkage analyses. Am J Hum Genet. 2007;81(3):559-75. https://doi.org/10.1086/51 9795.

Qu XQ, Hou BH, Sosso D, Osorio S, Fernie AR, Frommer WB. Sucrose efflux mediated by SWEET proteins as a key step for phloem transport. Science. 2012;335(6065):207-11. https://doi.org/10.1126/science.1213351.

Rosyara UR, De Jong WS, Douches DS, Endelman JB. Software for Genome-Wide Association Studies in autopolyploids and its application to potato. Plant Genome. 2016;9(2)

Roumeliotis E, Kloosterman B, Oortwijn M, Lange T, Visser RGF, Bachem CWB. Down regulation of $\mathrm{StGA30x}$ genes in potato results in altered GA content and affect plant and tuber growth characteristics. J Plant Physiol. 2013; 170(14):1228-34. https://doi.org/10.1016/j.jplph.2013.04.003.

Sang Q, Pajoro A, Sun H, Song B, Yang X, Stolze SC, et al. Mutagenesis of a quintuple mutant impaired in environmental responses reveals roles for CHROMATIN REMODELING4 in the Arabidopsis floral transition. Plant Cell. 2020;32(5):1479-500. https://doi.org/10.1105/tpc.19.00992.

Shennan S, Downey SS, Timpson A, Edinborough K, College S, Kerig T, et al. Regional population collapse followed initial agriculture booms in midHolocene Europe. Nat Commun. 2013;4(1):2486. https://doi.org/10.1038/ ncomms3486.

Si Y, Sankaran S, Knowles NR, Pavek MJ. Potato tuber length-width ratio assessment using image analysis. Am J Potato Res. 2017;94(1):88-93. https:// doi.org/10.1007/s12230-016-9545-1.

Song B, Liu T, Shen Y, Dong L, Jing S, Xie C, et al. Phytochrome F plays critical roles in potato photoperiodic tuberization. Plant J. 2019;98(1):42-54. https:// doi.org/10.1111/tpj.14198

Song JQ, Bradeen JM, Naess SK, Raasch JA, Wielgus SM, Haberlach GT, et al. Gene $\mathrm{RB}$ cloned from Solanum bulbocastanum confers broad spectrum resistance to potato late blight. Proc Natl Acad Sci USA. 2003;100(16):9128-33. https:// doi.org/10.1073/pnas.1533501100.

Sozzani R, Lee TJ, Chu TM, Wolfinger RD, Cella R, Hanley-Bowdoin L, et al. Global analysis of Arabidopsis gene expression uncovers a complex array of changes impacting pathogen response and cell cycle during geminivirus infection. Plant Physiol. 2008;148(1):436-54. https://doi.org/10.1104/pp.108.121038.

Spartz AK, Lee SH, Wenger JP, Gonzalez N, Itoh H, Inzé D, et al. The SAUR19 subfamily of SMALL AUXIN UP RNA genes promote cell expansion. Plant J. 2012;70(6):978-90. https://doi.org/10.1111/j.1365-313X.2012.04946.x.

Spooner DM, McLean K, Ramsay G, Waugh R, Bryan GJ. A single domestication for potato based on multilocus amplified fragment length polymorphism genotyping. Proc Natl Acad Sci USA. 2005;102(41):14694-9. https://doi.org/1 0.1073/pnas.0507400102.

Sumitomo K, Shirasawa K, Isobe S, Hirakawa H, Hisamatsu T, Nakano Y, et al. Genome-wide association study overcomes the genome complexity in autohexaploid chrysanthemum and tags SNP markers onto the flower color genes. Sci Rep. 2019;9(1):13947. https://doi.org/10.1038/s41598-019-50028-Z.

Tao Y, Zhao X, Wang X, Hathorn A, Hunt C, Cruickshank AW, et al. Large-scale GWAS in sorghum reveals common genetic control of grain size among cereals. Plant Biotechnol J. 2020;18(4):1093-105. https://doi.org/10.1111/ pbi.13284.

Willems A, Heyman J, Eekhout T, Achon I, Pedroza-Garcia JA, Zhu T, et al. The cyclin CYCA3;4 is a postprophase garget of the APC/C CCS52A2 E3-ligase controlling formative cell divisions in Arabidopsis. Plant Cell. 2020;32(9):297996. https://doi.org/10.1105/tpc.20.00208.

Xu X, Pan S, Cheng S, Zhang B, Mu D, Ni P, et al. Genome sequence and analysis of the tuber crop potato. Nature. 2011;475(7355):189-94. https://doi.org/10.1 038/nature10158.

Yang X, Luo Z, Todd J, Sood S, Wang J. Genome-wide association study of multiple yield components in a diversity panel of polyploid sugarcane (Saccharum spp.). Plant Genome. 2020;13(1):e20006.

Yu GC, Smith DK, Zhu HC, Guan Y, Lam TTY. GGTREE: an R package for visualization and annotation of phylogenetic trees with their covariates and other associated data. Methods Ecol Evol. 2017;8(1):28-36. https://doi.org/1 0.1111/2041-210X.12628.

Zhang K, Wang XW, Cheng F. Plant polyploidy: origin, evolution, and its influence on crop domestication. Hortic Plant J. 2019;5(6):231-9. https://doi.org/10.101 6/j.hpj.2019.11.003. 
Zhang YC, Tian F. Methods of potato experiment. Chapter 4: Potato breeding experiment research method. China Agricultural Science and Technology Press. 2007:4:91-93.

Zhao X, Bushman BS, Zhang X, Robbins MD, Larson SR, Robins JG, et al. Association of candidate genes with heading date in a diverse Dactylis glomerata population. Plant Sci. 2017;265:146-53. https://doi.org/10.1016/j.pla ntsci.2017.10.002

Zhu C, Gore M, Buckler ES, Yu JM. Status and prospects of association mapping in plants. Plant Genome. 2008;1(1):5-20. https://doi.org/10.3835/pla ntgenome2008.02.0089.

Zierer W, Rüscher D, Sonnewald U, Sonnewald S. Tuber and tuberous root development. Annu Rev Plant Biol. 2021;72(1):551-80. https://doi.org/10.114 6/annurev-arplant-080720-084456.

\section{Publisher's Note}

Springer Nature remains neutral with regard to jurisdictional claims in published maps and institutional affiliations.

Ready to submit your research? Choose BMC and benefit from:

- fast, convenient online submission

- thorough peer review by experienced researchers in your field

- rapid publication on acceptance

- support for research data, including large and complex data types

- gold Open Access which fosters wider collaboration and increased citations

- maximum visibility for your research: over $100 \mathrm{M}$ website views per year

At BMC, research is always in progress.

Learn more biomedcentral.com/submissions 